\title{
Evaluación de la intensidad del dolor y calidad de vida del paciente con diagnóstico de osteoporosis y tratamiento con teriparatida
}

\author{
Evaluation of the pain intensity of pain and quality of life of patients with \\ osteoporosis diagnosis and teriparatide treatment
}

\author{
Alejandro Pinzón Tovar' , Eduard Mauricio Díaz², Marcela Castro²
}

\section{Resumen}

Estudio descriptivo-retrospectivo para determinar si el tratamiento con hormona paratiroidea recombinante (teriparatida) reduce el dolor de los pacientes con osteoporosis, en el servicio de endocrinología del Hospital Militar de Colombia durante el año 2008

Objetivos: Describir aspectos demográficos de los pacientes que presentan osteoporosis y son manejados con teriparatida. 2) Determinar la frecuencia de los pacientes que requirieron terapia analgésica adyuvante para el manejo del dolor asociado a osteoporosis. 3) Determinar si el uso del teriparatida reduce el dolor de los pacientes con osteoporosis.

Materiales y métodos: De veinte pacientes con osteoporosis que recibían teriparatida, se incluyeron 12 para el estudio en un periodo de enero a diciembre del 2008. Para evaluar la calidad de vida y la intensidad del dolor, se usó el cuestionario SF36 y la escala visual análoga de dolor, respectivamente. Todos los datos proveniente de las encuestas y el formulario SF36, fueron almacenados en bases de datos usando el programa Excel 2011. Se realizó análisis estadístico descriptivo, usando la representación de frecuencias en forma de porcentaje. Para determinar diferencias en la frecuencia de la presentación de un evento, se usó la prueba de $\mathrm{Chi}^{2}$. Se tomó como significativo una $P<0.05$.

Resultados: El tratamiento con teriparatida disminuyó significativamente la frecuencia de terapia analgésica adyuvante con aines y opiáceos a los 6 meses de seguimiento $\left(P=0,04, \mathrm{Chi}^{2}\right)$.

Conclusiones: Los resultados aquí presentados sugieren un efecto benéfico de reducción del dolor y calidad de vida con el tratamiento para osteoporosis con teriparatida.

\section{Abstract}

A descriptive, retrospective study to determine whether treatment with recombinant parathyroid hormone (teriparatide) reduces painin patients with osteoporosis, in the endocrinology unit at the Military Hospital in Colombia in 2008.

Objectives: 1) to describe demographic aspects of osteoporosis patients managed with teriparatide. 2) to determine frequency of patients requiring analgesic adjuvant therapy for the management of pain associated with osteoporosis. 3) to determine whether the use of teriparatide reduced pain in patients with osteoporosis.

Materials and Methods: From twenty patients with osteoporosis who received teriparatide, 12 were included in the study in a period from January to December 2008. To evaluate the quality of life and pain intensity, the SF36 questionnaire and visual analog pain scale was used, respectively. All datafrom the survey sand the SF36 form, were store din databases using the program Excel 2011. A descriptive statistical analysis was performed using the frequency representation as a percentage. To determine differences in the frequency of occurrence ofan event, $\mathrm{Chi}^{2}$ test was used. $\mathrm{P}<0.05$ was considered as significant.

Results: Teriparatide treatment significantly decreased frequency of patients requiring analgesic adjuvant therapy with NSAIDs and opiates at 6 months follow-up ( $P=0.04, \mathrm{Chi}^{2}$ ).

Conclusions: results suggesta beneficial effect of pain relief and quality of life with treatment for osteoporosis with teriparatide.

Keywords: Pain, teriparatide, osteoporosis.

Palabras clave: Dolor, teriparatida, osteoporosis.

1 Médico internista, Universidad Surcolombiana Neiva. Endocrinólogo, Universidad Militar Nueva Granada - Hospital Militar de Colombia.

2 Residente de III año Medicina Interna, Universidad Surcolombiana Neiva.

Recibido: 08/10/2013 - Revisado: 03/03/2014 - Aceptado: 25/010/2014 
R.F.S Revista Facultad de Salud

Julio - Diciembre de 2014;6(2): 45-49
Evaluación de la intensidad del dolor y calidad de vida del paciente con diagnostico de osteoporosis y tratamiento con teriparatida / Alejandro Pinzón Tovar, et. al.

\section{Introducción}

La osteoporosis es una enfermedad sistémica de etiopatogénesis multicausal, caracterizada por baja densidad mineral y deterioro estructural del tejido óseo que lleva a fragilidad de los huesos e incremento en la susceptibilidad a sufrir fracturas. Los sitios más comprometidos son la cadera, la columna y la muñeca. ${ }^{[1,2]}$

Actualmente, 44 millones de personas se encuentran en riesgo de sufrir osteoporosis y de acuerdo al Instituto de Salud de los Estados Unidos, la osteoporosis es responsable de más de 1,3 billones de fracturas de cadera, columna y antebrazo cada año, más de la mitad de los casos ocurren en mujeres postmenopáusicas ${ }^{[3,4]}$. La morbilidad y la mortalidad asociada con fractura de cadera es alta en los individuos adultos mayores. ${ }^{[13]}$

Los tratamientos más comunes para la osteoporosis incluyen la suplencia de vitamina $D$, la administración de calcio y de bifosfonatos, que inhiben la pérdida ósea mediada por los osteoclastos, reduciendo el recambio óseo.

Recientemente, se ha empezado a usar en el tratamiento de la osteoporosis, la forma recombinante de la hormona paratiroidea humana (hPTH [1-34]), teriparatida ${ }^{\mathbb{E}}$; proteína que estimula la formación ósea. La hormona recombinante, gracias a sus propiedades anabólicas, también reduce el riesgo de fracturas en mujeres postmenopáusicas con osteoporosis. ${ }^{\left[{ }^{5-7]}\right.} \mathrm{A} 1$ parecer la aplicación en pulsos de ella, estimula la producción de osteoprotegerina por los osteoblastos e inhibe la síntesis del receptor activador del ligando nuclear del factor kappa (RANKL), el cual es necesario para la diferenciación de osteoclastos. ${ }^{[8]}$

El aumento de la densidad ósea se mantiene en mujeres con osteoporosis que reciben un esquema donde teriparatida se administra al inicio y posteriormente un bisfosfonato. El incremento de la densidad ósea vertebral, es considerablemente mayor con este tipo de terapia secuencial que con la administración de bisfosfonatos o estrógenos solos. ${ }^{[9,10]}$. Algunos estudios han planteado que alendronato altera la habilidad de teriparatida, disminuyendo el incremento de la formación ósea que se observa cuando se usa la hormona paratiroidea recombinante como monoterapia. ${ }^{[11,12]}$

Algunos efectos adversos relacionados con teriparatida incluyen: angina pectoris, hipercalcemia, hipertensión, hiperuricemia y sincope. Sin embargo, no existe incremento en el desarrollo de gota, artralgias o urolitiasis durante los estudios. La hipotensión ortostática transitoria no obliga a suspender el uso del medicamento y la elevación en los niveles de calcio no fue asociada con evolución adversa en pacientes que se mantuvieron asintomáticos. ${ }^{[14,15]}$ teriparatida no debe ser prescrito a pacientes con riesgos para desarrollar osteosarcoma, en este grupo se incluyen: enfermedad de Paget del hueso, elevación no explicada de fosfatasa alcalina ósea, niños y adultos jóvenes con epífisis abiertas o en quienes hayan recibido radioterapia ósea previa. ${ }^{[14,15]}$

El deterioro de calidad de vida que presentan los pacientes fracturados hace que teriparatida sea una terapia a consideran en osteoporosis relacionada al uso crónico de glucocorticoides con muy alto riesgo de fractura. ${ }^{[14]}$ Este medicamento representa una opción para el tratamiento de la osteoporosis con evidencia en disminución del riesgo de fractura. ${ }^{[11,16]}$

Aunque el efecto benéfico de teriparatida en el aumento de la densidad ósea en pacientes con osteoporosis ha sido previamente notado, su resultado sobre la intensidad del dolor que sufren estos pacientes no ha sido completamente documentada, más aun en pacientes locales. Nevitt y cols., mostraron una reducción del $61 \%$ en la incidencia del dolor en los pacientes tratados con teriparatida, durante el tratamiento y un seguimiento de 30 meses ${ }^{[18]}$. Un estudio Canadiense, publicado por Ali $y$ Cols., utilizando el "Mini-Osteoporosis Quality of Life Questionnaire" (OQLQ), estableció que después de varios meses de tratamiento con teriparatida se obtuvo mejoría en síntomas relacionados con el dolor funcionamiento emocional actividad física y estado de salud relacionado a la calidad de vida. En este trabajo se evaluó el efecto de la terapia con teriparatida en un grupo de pacientes con osteoporosis seguidos clínicamente durante un periodo de 12 meses.

\section{Metodología}

\section{Pacientes y diseño del estudio}

Este es un estudio descriptivo - retrospectivo que se acoge a la norma 8430 de 1993 en cuanto a las disposiciones académicas y éticas de investigación en salud. La unidad de análisis la componen los pacientes del Hospital Militar Central de Colombia, mayores de 18 años, con diagnóstico de osteoporosis por clínica y densitometría ósea (DXA), en tratamiento ambulatorio con hormona paratiroidea recombinante (teriparatida) hasta el año 2008. De veinte pacientes con osteoporosis que recibían teriparatida, en seis no correspondía el número telefónico con el nombre y dos rechazaron su participación en el estudio, por lo tanto los pacientes incluidos durante este periodo de tiempo fueron 12

\section{Evaluación de la calidad de vida y la intensidad del dolor}

El contacto inicial se realizó vía telefónica para concretar una cita en la que se explicó el desarrollo del estudio. Con los participantes se diligenció el consentimiento informado, la encuesta de recolección de datos y el cuestionario SF 36 . Este formato ha sido extensamente usado en estudios que evalúan la calidad de vida. Este formato estándar, valoró variables como edad, sexo, historia de tabaquismo y procedencia y otros. Además se tomó información sobre: fecha de inicio de tratamiento con teriparatida, meses de tratamiento

La valoración de dolor se realizó usando escala visual análoga numérica de 0 a 10 antes del inicio del fármaco, a los 6 meses y a los doce meses después del tratamiento; dosis diaria de analgésicos, antes del inicio del medicamento, a los 6 meses y a los 12 meses después; presencia de fracturas relacionadas a osteoporosis; localización de fractura; dolor actual en el sitio de fractura; necesidad de tratamiento quirúrgico para la fractura; tratamientos previos para osteoporosis y uso de aparatos ortésicos previo al tratamiento y a los doce meses de iniciar el medicamento.

El cuestionario SF-36 se aplicó a todos los pacientes incluidos durante entrevista personalizada, realizada por un entrevistador experimentado. En todos los casos la información fue suministrada por los pacientes directamente.

Toda la información fue tabulada y bases de datos usando el programa Excel 2011 fueron creadas.

\section{Análisis estadístico}

El análisis fue realizado con estadística descriptiva. Los datos son mostrados como $\mathrm{n}$ y $\%$ de $\mathrm{n}$ con respecto al total. Para evaluar diferencias en la frecuencia con que se presenta un evento (como 
paciente con mejoría con el tratamiento instaurado), se usó la prueba exacta de $\mathrm{Chi}^{2}$. Diferencias significativas fueron asumidas si la $\mathrm{P}$ fue $<0,05$.

\section{Resultados}

\section{Características sociodemográfica de los pacientes incluidos}

En este estudio, se evaluaron 12 pacientes con osteoporosis en tratamiento con teriparatida al inicio del mismo y a los 6 y 12 meses de instaurada la terapia. El promedio de edad de los pacientes incluidos fue de 68,8 años, la mayoría fueron mujeres postmenopáusicas, poco más de la mitad negaron exposición al cigarrillo y dos pacientes no reportaron fractura relacionada a osteoporosis (Tabla 1). Todos recibieron tratamiento con teriparatida mínimo un año como fue descrito en la sección de Materiales y Métodos

\section{Disminución del dolor en pacientes con osteoporosis y tra- tamiento con teriparatida}

Respecto al dolor, todos los pacientes, independiente de la edad, antes de iniciar el tratamiento con teriparatida manifestaron dolor de leve a severo; diez $(83,3 \%)$ manifestaron que el dolor era moderado o severo. E1 33,3\% de los pacientes que a los doce meses de tratamiento manifestaron un dolor menor a 5 en la escala visual análoga de dolor; eran mayores de 75 años. Durante el primer año de tratamiento con teriparatida, el $83,3 \%$ reportaron que la intensidad del dolor relacionado a osteoporosis disminuyó $(\mathrm{P}=0.012$, $\mathrm{Chi}^{2}$ test) (Tabla 2). En conclusión, estos resultados soportan el efecto benéfico de teriparatida en el tratamiento del dolor en pacientes con osteoporosis

\section{Medicamentos adicionales usados en la cohorte de pacientes}

Los analgésicos utilizados ambulatoriamente fueron anti inflamatorios no esteroideos y opiáceos (tramadol). Antes de iniciar el tratamiento con teriparatida, solo un paciente no tomaba ninguno de estos fármacos diariamente. A los seis meses de seguimiento, la mitad de los pacientes manifestaron no necesitar ningún analgésico de uso diario (Tabla 3).

Al culminar un año de tratamiento con teriparatida los pacientes usaban en promedio 0.5 (DS 0,9) analgésicos.

E1 $58,3 \%$, siete pacientes, habían recibido tratamiento antirresortivo previo y en este grupo se incluyen dos pacientes
Tabla 1. Características Socio-demográficas de la muestra de estudio.

\begin{tabular}{lc} 
Variable & $\mathbf{n}(\%)$ \\
Edad & \\
Media (DS) & $68,8(10,6)$ \\
Menor de 75 años & $8(66,6)$ \\
Mayores de 75 años & $4(44,4)$ \\
\hline
\end{tabular}

\section{Sexo}

Femenino

masculino

\section{Tabaquismo}

No fumadores

$5(41,6)$

Exfumador

$7(58,3)$

Fractura relacionado con Osteoporosis

$10(83,3)$

NO

$2(16,6)$

sin fractura que recibieron previamente bisfosfonatos. Todos, reportaron la presencia de dolor de leve a severo antes de iniciar teriparatida, seis $(85,7 \%)$ dijeron que el dolor era moderado o severo; pero al año de estar usando el medicamento, cinco $(71,4 \%)$ reportaron que su dolor era leve o no tenían dolor. De los cinco pacientes que no habían recibido tratamiento previo para osteoporosis, ninguno reporto dolor severo luego de doce meses de recibir teriparatida. Tabla 4.

\section{Tratamiento con teriparatida mejora calidad de vida en pacientes con osteoporosis}

Para valorar la calidad de vida se utilizó el cuestionario SF 36 que se diligencio durante la entrevista personalizada. El paciente suministro la información y no se presentaron datos incompletos. El cuestionario está conformado por ocho dominios que se valoran en relación a la media de cada dominio.

Al final del primer año de tratamiento con teriparatida, el $58,3 \%$ de los pacientes que reportaron un dolor menor a 5 en la escala visual análoga de dolor, manifestaron en el dominio dolor, vitalidad, función física y salud mental un valor mayor o igual a 50.

Tabla 2. Nivel de dolor en pacientes y tiempo tratamiento según edad.

\begin{tabular}{|c|c|c|c|c|c|c|}
\hline \multirow[b]{2}{*}{ Dolor: EVAD* } & \multicolumn{2}{|c|}{ Pretratamiento } & \multicolumn{2}{|c|}{180 días } & \multicolumn{2}{|c|}{360 días } \\
\hline & $\mathbf{n}$ & $\%$ & $\mathrm{n}$ & $\%$ & $\mathrm{n}$ & $\%$ \\
\hline Ninguno: $(0)$ & 0 & 0 & 2 & 16,6 & 3 & 25 \\
\hline Leve: (1 a 4) & 2 & 16,6 & 2 & 16,6 & 6 & 16,6 \\
\hline Moderado: (5 a 8) & 7 & 33,3 & 7 & 25 & 1 & 8,3 \\
\hline Severo: $(9$ a 10$)$ & 3 & 16,6 & 1 & 8,3 & 2 & 16,6 \\
\hline
\end{tabular}

* EVAD Escala visual análoga de dolor. 
R.F.S Revista Facultad de Salud Julio - Diciembre de 2014;6(2): 45-49
Evaluación de la intensidad del dolor y calidad de vida del paciente con diagnostico de osteoporosis y tratamiento con teriparatida / Alejandro Pinzón Tovar, et. al.
Tabla 3. Dosis diaria de analgésicos según tiempo de tratamiento.

\begin{tabular}{lccc} 
& Pretratamiento & 180 días & 360 días \\
Dosis & $\mathrm{n}(\%)$ & $\mathrm{n}(\%)$ & $\mathrm{n}(\%)$ \\
\hline 0 & $1(8,3)$ & $6(50)$ & $7(58,3)$ \\
1 & $8(66,6)$ & $4(33,3)$ & $4(33,3)$ \\
2 & $1(8,3)$ & $1(8,3)$ & 0 \\
3 & $2(16,6)$ & $1(8,3)$ & $1(8,3)$ \\
\hline
\end{tabular}

Con esta misma calificación del dolor, al final de los 12 meses de tratamiento; el 41,6\% de los participantes, manifestó en el dominio desempeño físico un valor menor que 50 , pero en los dominios desempeño emocional y salud general, el $66,6 \%$ registró un valor mayor o igual a 50; al igual que un $75 \%$ en el dominio función social. Tabla 5.
Tabla 4. Dolor y tiempo de tratamiento según tratamiento previo.

\begin{tabular}{|c|c|c|}
\hline & Dolor (EVAD*) & Tratamiento previo \\
\hline Pretratamiento & $\begin{array}{c}\text { SI } \\
\text { n (\%) }\end{array}$ & $\begin{array}{c}\text { NO } \\
\text { n (\%) }\end{array}$ \\
\hline Ninguno & 0 & 0 \\
\hline Leve (1 a 4$)$ & $1(8,3)$ & $1(8,3)$ \\
\hline Moderado (5 a 8) & $4(33,3)$ & $3(25)$ \\
\hline Severo $(9$ a 10$)$ & $2(16,6)$ & $1(8,3)$ \\
\hline \multicolumn{3}{|c|}{360 Días de tratamiento con teriparatida } \\
\hline Ninguno & $1(8,3)$ & $2(16,6)$ \\
\hline Leve (1 a 4$)$ & $4(33,3)$ & $2(16,6)$ \\
\hline Moderado (5 a 8 ) & 0 & $1(8,3)$ \\
\hline Severo $(9$ a 10$)$ & $2(16,6)$ & 0 \\
\hline
\end{tabular}

* EVAD Escala visual análoga de dolor.

Tabla 5. Dominios de calidad de vida y dolor a los 12 meses de tratamiento.

\begin{tabular}{|c|c|c|c|c|c|}
\hline & $\begin{array}{l}\text { Dolor } \\
\text { EVAD* }\end{array}$ & $\begin{array}{c}\text { Ninaunn } \\
\text { (0) }\end{array}$ & $\begin{array}{l}\text { Leve } \\
\text { (1 a 4) }\end{array}$ & $\begin{array}{c}\text { Moderado } \\
\text { (5 a } 9)\end{array}$ & $\begin{array}{c}\text { Severo } \\
(9 \text { a } 10)\end{array}$ \\
\hline Salud general & $\begin{array}{l}<50 \\
\mathrm{n}(\%) \\
>50 \\
\mathrm{n}(\%)\end{array}$ & $\begin{array}{c}0 \\
3(25)\end{array}$ & $\begin{array}{c}1(8,3) \\
5(41,6)\end{array}$ & $\begin{array}{c}18,3) \\
0\end{array}$ & $\begin{array}{c}2(16,6) \\
0\end{array}$ \\
\hline Función física & $\begin{array}{l}<50 \\
\mathrm{n}(\%) \\
>50 \\
\mathrm{n}(\%)\end{array}$ & $\begin{array}{c}0 \\
3(25)\end{array}$ & $\begin{array}{l}2(16,6) \\
4(33,3)\end{array}$ & $\begin{array}{c}18,3) \\
0\end{array}$ & $\begin{array}{l}1(8,3) \\
1(8,3)\end{array}$ \\
\hline Desempeño físico & $\begin{array}{l}<50 \\
\mathrm{n}(\%) \\
>50 \\
\mathrm{n}(\%)\end{array}$ & $\begin{array}{c}0 \\
3(25)\end{array}$ & $\begin{array}{c}5(41,6) \\
1(8,3)\end{array}$ & $\begin{array}{c}1(8,3) \\
0\end{array}$ & $\begin{array}{c}2(16,6) \\
0\end{array}$ \\
\hline Desempeño emocional & $\begin{array}{l}<50 \\
\mathrm{n}(\%) \\
>50 \\
\mathrm{n}(\%)\end{array}$ & $\begin{array}{c}0 \\
3(25)\end{array}$ & $\begin{array}{c}1(83) \\
5(41,6)\end{array}$ & $\begin{array}{c}0 \\
1(8,3)\end{array}$ & $\begin{array}{c}2(16,6) \\
0\end{array}$ \\
\hline Dolor & $\begin{array}{l}<50 \\
\mathrm{n}(\%) \\
>50 \\
\mathrm{n}(\%)\end{array}$ & $\begin{array}{c}0 \\
3(25)\end{array}$ & $\begin{array}{l}2(166) \\
4(33,3)\end{array}$ & $\begin{array}{c}18,3) \\
0\end{array}$ & $\begin{array}{c}2(16,6) \\
0\end{array}$ \\
\hline Vitalidad & $\begin{array}{l}<50 \\
\mathrm{n}(\%) \\
>50 \\
\mathrm{n}(\%)\end{array}$ & $\begin{array}{c}0 \\
3(25)\end{array}$ & $\begin{array}{l}2(166) \\
4(33,3)\end{array}$ & $\begin{array}{c}1(8,3) \\
0\end{array}$ & $\begin{array}{c}2(16,6) \\
0\end{array}$ \\
\hline Salud mental & $\begin{array}{l}<50 \\
\mathrm{n}(\%) \\
>50 \\
\mathrm{n}(\%)\end{array}$ & $\begin{array}{c}0 \\
3(25)\end{array}$ & $\begin{array}{l}2(16,6) \\
4(33,3)\end{array}$ & $\begin{array}{c}0 \\
1(8,3)\end{array}$ & $\begin{array}{l}1(8,3) \\
1(8,3)\end{array}$ \\
\hline Función social & $\begin{array}{l}<50 \\
\mathrm{n}(\%) \\
>50 \\
\mathrm{n}(\%)\end{array}$ & $\begin{array}{c}0 \\
3(25)\end{array}$ & $\begin{array}{c}0 \\
6(50)\end{array}$ & $\begin{array}{c}0 \\
1(8,3)\end{array}$ & $\begin{array}{l}1(8,3) \\
1(8,3)\end{array}$ \\
\hline
\end{tabular}

* Escala visual análoga del dolor. 


\section{Discusión}

Todos los pacientes diagnosticados con osteoporosis eran mayores de 50 años y la mayoría fueron mujeres, aspectos que se relacionan con las publicaciones de la literatura mundial ${ }^{[17]}$. No se reportó fractura a nivel de cadera, pero podemos mencionar que el $40 \%$ de los pacientes fracturados tenían compromiso de hueso cortical (fractura de antebrazo) y el $60 \%$ de los pacientes tenían compromiso de hueso trabecular (fractura vertebral). Al parecer el efecto sobre dolor de teriparatida se presentó en ambos grupos independiente del tipo de hueso comprometido y de la necesidad de intervención quirúrgica.

Al finalizar el primer año, el 75\% de los pacientes reportaron dolor leve o ningún dolor relacionado a osteoporosis, al igual que en estudios previos parece existir algún efecto sobre el control del dolor durante el tratamiento con teriparatida. En el 2006, Nevitt demostró que la efectividad del fármaco en controlar el dolor no solo se da durante el tratamiento sino en un seguimiento a 30 meses luego de terminada la terapia. Su publicación determinó una reducción del $61 \%$ en la incidencia del dolor en los pacientes tratados con teriparatida ${ }^{[18]}$.

En nuestro estudio, podría decirse que el efecto sobre el dolor es independiente de la edad y es posible que tratamientos previos para osteoporosis no afecten la influencia de teriparatida sobre el dolor relacionado a osteoporosis. En los primeros 12 meses la mayoría de los pacientes $(83,3 \%)$ reportaron que la intensidad del dolor relacionado a osteoporosis había disminuido.

El uso de analgésicos disminuyo según lo reportado durante la entrevista luego del primer año de tratamiento. Manuele en el 2007. realizo un estudio donde los resultados demostraron que la terapia con teriparatida mejoró la calidad de vida y redujo el consumo de antiinflamatorios no esteroideos en un $65 \%{ }^{[19]}$.

Durante el diligenciamiento del cuestionario SF 36 que se realizó al final del estudio, los dominios de calidad de vida: salud general, función social, dolor, vitalidad, función física, desempeño emocional y salud mental presentaron una mediana mayor o igual a 50 con escala visual análoga de dolor luego de un año de tratamiento menor a 5; es lógico pensar que con menor dolor los dominios de calidad de vida obtengan un valor por encima de la media.

El presente estudio es un estudio de serie de casos con datos retrospectivos lo que representa una limitación para la interpretación epidemiológica de la información. La información retrospectiva proporciona un sesgo de memoria. El diligenciamiento del cuestionario de calidad de vida se hizo en el momento de realizar el estudio y no al inicio del tratamiento con teriparatida lo que no permite evaluar la calidad de vida previa al tratamiento. Por el tipo de estudio no se pueden concluir que los resultados sean significativos o representativos desde el punto de vista epidemiológico.

\section{Conclusiones}

El uso del teriparatida en pacientes con osteoporosis, redujo significativamente el dolor en ellos. Se encontró también una reducción en el uso de analgésicos debido al efecto benéfico de teriparatida en el tratamiento para osteoporosis y además las calificaciones de los dominios para calidad de vida dadas por el formulario SF36, estuvieron por encima de la media en la mayoría. A nivel regional, deben realizarse estudios futuros enfocados en demostrar la eficacia de teriparatida en la disminución del dolor relacionado a osteoporosis y la mejoría en la calidad de vida

\section{Referencias}

1. Warriner A, Saag K, Osteoporosis Diagnosis and Medical Treatment. Orthop Clin N Am 44. 2013:125-135.

2. Rachner T, Khosla S, Hofbaver L, Osteoporosis: now and the future. Lancet $2011 ; 377: 1276-87$.

3. Cauley J, Public Health Impact of Osteoporosis. J Gerontol A Biol Sci Med Sci 2013;68(10):1243-1251.

4. Eriksen EF, Halse J, Moen MH. New developments in the treatment of osteoporosis. Acta Obstet Gynecol Scand 2013;92:620-636.

5. Cosman F, Lane NE, Bolognese MA, et al. Effect of transdermal teriparatide administration on bone mineral density in postmenopausal women. J Clin Endocrinol Metab 2010;95:151 e8.

6. Appelman-Diikstra N, Papapoulos S, Novel approaches to the treatment of osteoporosis. Best Pract Res Clin Endocrinol Metab 28. 2014:843-857.

7. Pinkerton J, Thomas S, Dalkin A, Osteoporosis Treatment and Prevention for Postmenopausal Women: Current and Future Therapeutic Options. Clin Obstet Gynecol. 2013;56(4):711-721.

8. Baron R, Hesse E, Update on Bone Anabolics in Osteoporosis Treatment:Rationale, Current Status, and Perspectives. J Clin Endocrinol Metab, 2012;97:31 1-325.

9. Tella S, Gallagher JC, Prevention and treatment of postmenopausal osteoporosis. J Steroid Biochem Mol Biol 142. 2014:155-170.

10. Cosman F. Combination therapy for osteoporosis: a reappraisal. BoneKEy Rep 2014: 3. Article No. 518.

11. Augustine $M$, Horwitz $M$, Parathyroid Hormone and Parathyroid Hormone-Related Protein Analogs as Therapies for Osteoporosis. Curr Osteoporos Rep, 2013;1 1:400-406.

12. Finkelstein, J. S. Effects of teriparatide, Alendronate, or Both on Bone Turnover in Osteoporotic Men. J Clin Endocrinol Metab 2006;91:2882-2887.

13. Modi A, Sajian S, Gandhi S. Challenges in implementing and maintaining osteoporosis therapy. International Journal of Women's Health 2014;6:759-769.

14. Uihlein A, Leder B, Anabolic Therapies for Osteoporosis. Endocrinol Metab Clin N Am 41, 2012; pp. 507-525.

15. Tella S, Gallagher J, Biological agents in management of osteoporosis. Eur J Clin Pharmacol, 2014;70:12911301.

16. Das S, Crockett J, Osteoporosis - a current view of pharmacological prevention and treatment. Drug Design, Development and Therapy 2013;7:435-448.

17. Hernlund $E$, Svedbom A, lvergârd $M$, Compston J, Cooper C, Stenmark J, "etc". Osteoporosis in the European Union: medical management, epidemiology and economic burden. Arch Osteoporos, 2013;8:136.

18. M. C. Nevitt. Reduction in the risk of developing back pain persists at least 30 months after discontinuation of teriparatide treatment: a meta-analysis. Osteoporos Int, 2006; 17:1630-1637.

19. Manuele, S. The teriparatide in the treatment of severe senile osteoporosis. Arch. Gerontol. Geriatr. 2007; 1:249-258. 